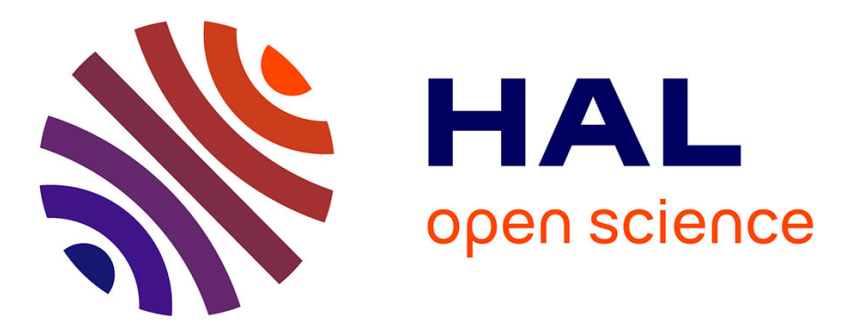

\title{
Measurement of tritium in the free water of milk: spotting and quantifying some biases and proposing ways of improvement
}

Pierre Le Goff, Jean-Marie Duda, Philippe Guétat, Pauline Rambaud, Christophe Mavon, Laurent Vichot, Pierre-Marie Badot, Michel Fromm

\section{To cite this version:}

Pierre Le Goff, Jean-Marie Duda, Philippe Guétat, Pauline Rambaud, Christophe Mavon, et al.. Measurement of tritium in the free water of milk: spotting and quantifying some biases and proposing ways of improvement. Journal of Environmental Radioactivity, 2014, 127, pp.1-10. 10.1016/j.jenvrad.2013.09.006 . hal-01117741

\author{
HAL Id: hal-01117741 \\ https://hal.science/hal-01117741
}

Submitted on 17 Feb 2015

HAL is a multi-disciplinary open access archive for the deposit and dissemination of scientific research documents, whether they are published or not. The documents may come from teaching and research institutions in France or abroad, or from public or private research centers.
L'archive ouverte pluridisciplinaire HAL, est destinée au dépôt et à la diffusion de documents scientifiques de niveau recherche, publiés ou non, émanant des établissements d'enseignement et de recherche français ou étrangers, des laboratoires publics ou privés. 
4 Pierre Le Goff ${ }^{1,2,3}$, Jean-Marie Duda ${ }^{1}$, Philippe Guétat ${ }^{1,4}$, Pauline Rambaud ${ }^{1}$, Christophe

5 Mavon $^{2}$, Laurent Vichot ${ }^{1}$, Pierre-Marie Badot ${ }^{3}$, Michel Fromm $^{3}$

6

$7 \quad{ }^{1}$ : CEA Valduc, 21120 Is-sur-Tille - France

$8{ }^{2}$ : UMR CNRS 6249 Chrono-Environnement / LCPR-AC, Université de Franche-Comté, 16 route de Gray.

925030 Besançon Cedex - France

10

3 :UMR CNRS 6249 Chrono-Environnement, Université de Franche-Comté, 16 route de Gray.

1125030 Besançon Cedex - France

12

${ }^{4}: \mathrm{CEA} / \mathrm{HC}$

13

Corresponding author:

pierre.legoff@ cea.fr - +33380234000 - +33380235209

\section{Abstract}

As one of the three natural isotopes of hydrogen, tritium is ubiquitous and might potentially be present in any water or organic molecule that constitutes a biological matrix. Milk is one of the most frequently monitored foodstuffs in the vicinity of chronic release of radionuclides, as it is a very common product and also because it integrates deposition on large areas of grass at a local scale. Different parameters have been studied to assess their impact on the reliability of tritium measurements. The volume of the sample, the technique used to extract the water and the level of dehydration modulate the results but in different ways: dispersion of results, under- or overestimation of the tritium activity. The influence of sample storage and preparation has also been investigated. Methodological improvements of tritium measurements in the free water of milk are proposed.

\section{Key words}

30 Tritium measurement

31 Free water

32 Isotopic fractionation 
33 Environment

34

\section{Highlights}

36 Biases in tritium assay are caused by the conditions in which the water is extracted

37 Isotopic fractionation does not fit with the Rayleigh formula when milk is distilled

38 Recommendations are made to improve tritium activity measurement 
Introduction

Among the unstable isotopes released by the nuclear industry, the quantities of tritium reaching the environment are usually small and generally fit easily with regulatory limits. As tritium was massively released during the atmospheric nuclear tests between 1945 and1980, it has become widely dispersed in the environment and in food chains. Its quantity in the atmosphere peaked in 1963 and has been decreasing ever since. It is now mainly localized in the water of oceans (about $99 \%$ ) (Jacobs, 1968; Weaver et al., 1969)(UNSCEAR, 2008). Nevertheless, tritium, along with ${ }^{14} \mathrm{C}$ and noble gases remain the dominant radionuclides released into the atmosphere by the nuclear industry. The main anthropic sources are weapon facilities, nuclear power plants, reprocessing facilities, the production and use of labelled compounds for medical use, research or even self-powered lighting products and research facilities for nuclear fusion (Guétat et al., 2008; IRSN/DEI, 2010).

Being an isotope of hydrogen, tritium can be incorporated into almost all components of biological systems: water (HTO) or organic molecules (Diabaté and Strack, 1993) (so-called Organically Bound Tritium or OBT). When dealing with OBT, two categories of atomic bonds are generally distinguished: 

bound tritium is known as non-exchangeable Organically Bound Tritium (neOBT).

Exposure of individuals depends on the type of the tritiated molecule(s) incorporated as well as on its/their metabolism. When tritium originates from tritiated water release and is further integrated in the food chain by, for example, going through photosynthesis (see further details in Boyer et al., 2009), some simplifications are considered to define a single "dose per unit intake factor" (ICRP 1989, 1997):

- considering exchangeable/non exchangeable proportions to be equal, - $\quad$ considering an average biological half-life of 40 days for all non-exchangeable tritium of all organic molecules.

When using liquid scintillation counting, measurements of tritium specific activity on water allow the lowest decision threshold to be reached. Laboratories that measure tritium in environmental samples frequently use a same protocol:

- $\quad$ extraction of the free water of the sample and measure (Free Water Tritium), then

- $\quad$ on the one hand: oxidisation of the dry fraction resulting in the production of combustion water, then measurement of total organic tritium (i.e. the sum of eOBT and neOBT),

- $\quad$ on the other hand: isotopic exchange of hydrogen isotopes by washing the dry fraction with tritiated water, thus a second extraction of water to measure (if possible) eOBT and oxidisation of the "washed" and dried fraction to measure the neOBT as combustion water. 
Frequently, eOBT is not measured but deduced from the following simple relation:

$$
\mathrm{eOBT}=\mathrm{OBT}-\mathrm{neOBT}
$$

$$
\text { Every isotope or inaccuracy effect in every step of the procedure may induce errors in the }
$$
measurement of the specific activity of extracted free water and of OBT (Baumgärtner and Kim, 1990; Kim and Baumgärtner, 1991).

Usually, water is extracted from fresh samples or after isotopic rinsing by at least one of the following four techniques: (1)

- $\quad$ filtration: it allows quick and easy recovery of the main part of the dry matter, except soluble molecules which are in the filtrate. The bias induced depends on the filtration technique (i.e. characteristics of the filter) and on the nature of the sample. Retentate and distillate both need further treatment prior to measurement.

- distillation: it is performed under atmospheric pressure or under reduced pressure, it allows the recovery of almost pure water. Under reduced pressure, it is possible to completely distil at lower temperature (which induces less degradation of organic samples), to prevent the risk of contamination of extracted water by pyrolitic products (Wood et al., 1993) and to limit the isotopic effect during evaporation.

- $\quad$ azeotropic distillation extracts water at lower temperatures than distillation. As it uses organic compounds, it is more difficult to perform and it can additionally induce contamination of the dry matter by hydrocarbons. 
- $\quad$ Freeze drying: i.e. extraction of water via sublimation; it has the same advantages as distillation under reduced pressure. The size of the apparatus, the temperature of the cold trap (usually $>-20^{\circ} \mathrm{C}$ ) and the time required to completely extract the water may induce biases by condensing atmospheric vapour before starting or during the process.

120 Repetition of measurements performed in our laboratory on the free water of a given tritiated milk obtained by distillation under reduced pressure or by freeze-drying, have shown certain systematic errors and dispersion of the values beyond the basic uncertainties of the measurements. The reasons underlying these differences have been sought and improvements

124 of the reliability of tritium measurements are proposed.

Four possible hypothetical origins of the observed differences in measured specific activities were identified: measurement.

- $\quad$ the influence of the mass of sample: as each water removal technique has a specific dead volume and a specific geometry, the global yield of dehydration 
can be influenced and thus be the origin of a bias in the measurement of specific activity. the influence of the final degree of dehydration: if isotopic fractionation occurs during water removal, the final level of dehydration will influence the specific activity measured.

\section{Materials and Methods}

146 Water was extracted from nineteen aliquots of the same milk sample (collected in the vicinity

147 of the Valduc Centre of the French Atomic Agency) using three different dehydration

148 techniques. The experiments were completed with twenty-one measurements performed on

149 milks collected for our routine activity. Each time, weights of fresh milk, of dry matter and of 150 collected water were noted.

\subsection{Analytical method}

154 Specific tritium activities were measured by liquid scintillation counting (PerkinElmer Tri-

155 Carb 2910 TR) with an overall precision $(2 \sigma)$ of $\pm 17 \%$. The scintillator used was Ultimagold 156 LLT (Packard). Quenching effects of the measuring system were carefully examined and the 157 results measured corrected accordingly.

\subsection{Storage of samples}

161 The commercially available source water Volvic is considered to have very low levels of

162 tritium. It is commonly used in laboratories as a blank. In order to check if storage of samples 
163 in Valduc induced biases in the measurement of the specific activity, samples of Volvic water

164 were stored in different conditions and their specific activity was been measured after $6 \mathrm{~h}, 16$

165 h, 24 h, 48 h, 96 h, 1 week, 2 weeks, 3 weeks or 30 days of storage.

166

167 Modifications in the conditions of storage were performed to test the influence of the

168 temperature and the type of bottle in which the samples were stored.

169

170 Four sets of nine samples of $50 \mathrm{~mL}$ of Volvic water were stored in $150 \mathrm{~mL}$ polyethylene

171 bottles at $-25^{\circ} \mathrm{C}, 3^{\circ} \mathrm{C}, 20^{\circ} \mathrm{C}$ and $40^{\circ} \mathrm{C}$.

172

173 Five other sets of nine samples of Volvic were stored in different kind of bottles: $50 \mathrm{~mL}$ of Volvic water in $150 \mathrm{~mL}$ high density polyethylene (HDPE) bottles, $150 \mathrm{~mL}$ of Volvic water in the same kind of bottles, $50 \mathrm{~mL}$ of Volvic water in the same bottles placed in double welded vinyl bags, $20 \mathrm{~mL}$ of Volvic water in $20 \mathrm{~mL}$ glass bottles, $20 \mathrm{~mL}$ of Volvic water in $20 \mathrm{~mL}$ HDPE bottles (usually used for scintillation counting)

$1831 \mathrm{~L}$ of Volvic water was also stored in an open $1.5 \mathrm{~L}$ bottle. $10 \mathrm{~mL}$ were sampled after $6 \mathrm{~h}, 16$ 184 h, 24 h, 48 h, 96 h, 1 week and 2 weeks of storage. This experiment was shorter than the 185 others since there was no water left in the bottle after the seventh sampling (due to 186 evaporation and aliquot removal). 


\subsection{Techniques of water removal}

\subsubsection{Comparison of common techniques}

192 Three commonly used techniques of dehydration were compared pairwise: condenser - $\quad$ freeze drying using a Heto Drywinner PL3000.

Equal quantities of samples were used in each group of paired samples. In the first technique, the sample was introduced in a $1 \mathrm{~L}$ Erlenmeyer flask in a water bath at $55^{\circ} \mathrm{C}$. The flask was fitted with a splash head (to prevent or limit the sample from spurting in the apparatus during distillation). The distillate was collected from a Liebig condenser containing a flow of $3^{\circ} \mathrm{C}$ thermostated water. Its dead volume was $0.69 \mathrm{~mL} \pm 0.25 \mathrm{~mL}$. The distillation bridge was connected to a Vacuubrand ME 2C pump working at full capacity. The condensate was recovered in an Erlenmeyer also at in a water bath at $3^{\circ} \mathrm{C}$. At the end of dehydration, the first water bath was heated to $70{ }^{\circ} \mathrm{C}$.

Distillation under reduced pressure was also conducted using rotating evaporator. This technique differs from the previous one by the apparatus used. One of the major differences

210 between them is the geometry of the condenser: a rotating evaporator is equipped with a

211 diagonal spiral condenser which has a dead volume of about $7 \mathrm{~mL}$. Samples are introduced in

212 a $1 \mathrm{~L}$ flask which is then connected to a rotating evaporator (Buchi Rotavapor R200 or Buchi 
213 Rotavapor RE 121 equiped with Buchi 471 Oil bath) connected to a pump (Vacuubrand ME

214 2C) which is also used at its full capacity. Condensed vapours are recovered in a flat-

215 bottomed flask. As with the previous technique, the water bath was thermostated first at $55^{\circ} \mathrm{C}$

216 and then at $70^{\circ} \mathrm{C}$. The water flowing in the condenser was thermostated at $3^{\circ} \mathrm{C}$.

218 Freeze drying was performed with a modified Heto Drywinner PL 3000. Samples were

219 introduced in acrylic pots connected to a manifold which is connected to a glass insert. The

220 insert was placed in the cold trap of the Heto Drywinner PL 3000 (temperature: $-55^{\circ} \mathrm{C}$ ) to

221 allow the required decontamination of the cold trap between samples thus avoiding "memory

222 effects". The manifold was also connected to the pump (Adixen Pascal 1005) used for general

223 vacuum applications. Pressure in the system was $<0.5 \mathrm{hPa}$. After complete dehydration of the

224 samples, the glass insert was removed from the system and immediately sealed to avoid

225 contamination of the extracted water with atmospheric moisture until the ice has completely

226 thawed.

227 Dehydration techniques were compared by pairwise to improve the power of the statistic tests

228 (9 repetitions to compare freeze drying and rotating evaporator and 8 for freeze drying vs.

229 distillation bridge and 8 for rotating evaporator vs. distillation bridge).

\section{$230 \quad$ 1.3.2 Test of the reliability of freeze drying and distillation}

231 The specific activity of tritiated pure water (type 3 produced by RiOs 3 Water Purification

232 System (Merck Millipore)) were measured in three cases:

$233 \quad$ - $\quad$ without other treatment,

$234 \quad$ - $\quad$ after being distilled under reduced pressure using a distillation bridge (as

235 described above),

$236 \quad$ - $\quad$ after being freeze-dried (in the conditions described above). For each case, 3

$237 \quad$ aliquots of $49.9 \mathrm{~g} \pm 0.1 \mathrm{~g}$ were prepared. 


\subsubsection{Influence of ambient atmosphere on freeze drying}

To detect possible external contamination, two kinds of experiments were performed:

241 Measurement of the specific activity of water extracted by freeze drying in two different

242 ambient atmospheres: one in the Valduc Centre (in the conditions described above) and one in

243 Besançon (25-France) where the specific activity of the atmosphere in HTO is below the

244 decision threshold. The milk was separated into 7 samples of $51.23 \mathrm{~g} \pm 0.45 \mathrm{~g}$. They were

245 frozen in Valduc in plastic bottles inserted in double welded vinyl bags. Three were freeze-

246 dried in Valduc, three in Besançon and one was distilled using a distillation bridge in the

247 Valduc Centre in the conditions described above. Freeze drying at Besançon was performed

248 in a Cosmos 20k (Cryotec). Vaccum was generated by a pump (Adixen Pascal 2005-Ci)

249 working at full capacity. After the end of freeze drying, the water was recovered by heating

250 the condenser. The specific activity of each recovered water sample was measured in the

251 Valduc Centre and compared.

253 Empty freeze drying: Drywinner Heto PL 3000 was used empty three times for 5-7 days at

254 Valduc Centre. A commercial bubbling system (MARC 7000-SDEC France) was used to 255 monitor the atmospheric tritium levels during the third repetition. After the end of freeze 256 drying, the mass of the cold trap was measured and compared to its mass when empty. Then, $25710 \mathrm{~mL}$ of non-tritiated water was inserted into the cold trap to recover possible traces of water 258 trapped during freeze drying. The specific activity of the water in the cold trap was measured, 259 taking into account the dilution and compared to the specific activity of the water in the pots 260 of the bubbling system. 
264 To test the influence of the quantity of the sample on the reliability of measures, 6 masses

265 were considered (about 15, 30, 60, 120, 240 and $480 \mathrm{~g}$ ) and samples were treated by two of

266 the three previously presented methods: distillation under reduced pressure using a distillation

267 bridge or a rotating evaporator. Most of the sets were composed of two samples prepared with

268 the distillation bridge and one with the rotating evaporator. The central point at 30-38 $\mathrm{g}$ was

269 composed of three more samples treated with the rotating evaporator. The highest mass was

270 only composed of one measurement since in other repetitions milk spurted throughout the

271 apparatus until there was none left.

272

273

\subsection{State of dehydration}

274

275

The weight of fresh milk before treatment, and of dry matter and water after dehydration on the other hand, provide correlation between the mass of water extracted from milk and the 277 specific activity measured.

"Sequential distillations" of milk were performed. The apparatus used in these experiments is illustrated in Fig. 1. A sample of about $300 \mathrm{~mL}$ was introduced in a $1 \mathrm{~L}$ Erlenmeyer flask in a $55^{\circ} \mathrm{C}$ bath. The flask was connected to a splash head to prevent or limit the sample from spurting into the apparatus during distillation. When the system is under reduced pressure, vapours flow to a condenser at $3^{\circ} \mathrm{C}$ and connected to a Vacuubrand $\mathrm{ME} 2 \mathrm{C}$ pump working at full capacity. The condensed vapour then falls into a dropping funnel. Each 10-30 mL (23 mL on average), it is opened to let the water flow into a $50 \mathrm{~mL}$ Erlenmeyer. Once the dropping funnel is empty, it is closed to collect the next aliquot and the water is collected from the 50 mL Erlenmeyer and weighed. The experiment is pursued until the sample is completely dry. 
288 When possible, the dry matter of the sample is freeze-dried to collect any water which could 289 remain. The specific activity of each aliquot is measured.

290

291 Fig. 1: Apparatus used for "sequential distillations"

292

293 Sequential distillations were carried out on four different milks collected for our routine 294 measurements. 


\section{Results and discussion}

\subsection{Influence of samples storage}

298

299 Only one set of samples showed any significant change in its specific activity during storage:

300 the Volvic water stored in an open bottle (Fig. 2). The specific activity of the water increased

301 until it reached equilibrium with the atmospheric water vapour $\left(178.5 \pm 133.0 \mathrm{~Bq} \mathrm{~L}^{-1}\right)$ after

302 two weeks.

303

304 Fig. 2: Specific activity of Volvic water stored in an open bottle versus duration of storage. The first point 305 having a specific activity under the limit of detection was plotted as having a specific activity of $0 \mathrm{~Bq} \mathrm{~L}{ }^{-1}$.

307 All the other results of the experiments remained below the decision threshold $\left(2.8 \mathrm{~Bq} \mathrm{~L}^{-1}\right)$

308 during storage.

310 This proves that storage in well closed plastic or glass bottles is able to prevent the marking of

311 the samples from the laboratory environment even in the case of the relatively tritiated

312 atmosphere of a nuclear centre. Nevertheless, to avoid any cross contamination, we decided to

313 store samples at $-20^{\circ} \mathrm{C}$ (to preserve organic matter during storage) and in double packaging

314 (bottle + sealed vinyl pocket or double sealed vinyl pocket) to avoid any unintentional

315 marking. 


\subsection{Influence of the technique of dehydration}

\subsubsection{Comparison of three common techniques}

319 The specific activity of water extracted from milks using the three techniques previously described was measured (mean: $60.4 \mathrm{~g}$ ). The three techniques were not performed on each of the collected milk samples. Fig. 3 shows how the results are distributed.

Fig. 3: Comparison of the distributions of measured specific activities of water extracted using the three different methods described in this study. Central boxes represent the values from the lower to upper quartile. Middle lines represent the median. Vertical lines extend from the minimum to the maximum value of each population, which are represented by horizontal lines at their extremity. The specific activity of the water extracted by freeze drying is significantly higher than the specific activity of the water extracted by each of the two distillation methods tested (Wilcoxon test, $\mathrm{p}<0.01$ ). Differences between the two techniques of distillation were not significant.

On the one hand, both methods of distillation (distillation bridge and rotating evaporator) gave similar median concentrations but the rotating evaporator technique showed a standard deviation $22 \%$ higher than the distillation bridge. On the other hand, the measured specific activities of water extracted by freeze drying were $40 \%$ higher when compared to the results obtained with a rotating evaporator. Three experiments were performed to explain these results.

\subsubsection{Reliability of distillation and freeze drying}

339 The specific activity of pure water was measured with or without a complementary treatment

340 (i.e. distillation or freeze drying) performed in the Valduc Centre. The results of this

341 experiment are presented in Fig. 4. 
Fig. 4 : Comparison of specific activities measured on water with and without treatment (distillation or freeze drying)

346 Distillation had no significant incidence on the measured specific activity of extracted water $(+2.9 \%)$ whereas freeze drying led to a clear increase $(+42.9 \%)$.

\subsubsection{Influence of ambient atmosphere on freeze drying}

349 First, to test the effect of ambient air during freeze drying, two sets of three $50 \mathrm{~g}$-aliquots of

350 the same milk sample were freeze-dried, one set in Besançon and one in the Valduc Centre.

351 Freeze drying in both the Valduc Centre and Besançon led to almost complete extraction of

352 the water, i.e. $88 \%$ of the weight of the total sample without any significant difference

353 between samples (standard deviation: $0.09 \%$ ). Nevertheless, the comparison of the specific

354 activities of the two sets of samples did show significant differences (see Fig. 5).

Fig. 5 : Specific activities of water extracted from milk by means of freeze drying performed in Besançon and in

This difference can only be explained by a (de)marking of the extracted water by the condensation of atmospheric water vapour. This phenomenon can occur during freeze drying by leaks in the apparatus, or before freeze drying by condensation of atmospheric water on the frozen sample or even after freeze drying when the vacuum is broken to recover the condensed water. Note that the observed deviation after freeze drying in Besançon is high regarding the little difference of specific activities between atmospheric water $\left(<5.8 \mathrm{~Bq} \mathrm{~L}^{-1}\right)$ and free water of milk (about $20 \mathrm{~Bq} \mathrm{~L}^{-1}$ ).

The apparatus used in the Valduc Centre avoided the risk of exchange during melting of frozen condensate but this was not the case in Besançon. This can explain why the impact of condensed atmospheric vapour was so significant in Besançon whereas the differences in 
specific activities remained small. In laboratories specifically equipped for measurement of

371 tritium (for example with a small cold trap which can be isolated from the atmosphere while

372 the condensed water is recovered), the biases would be, at worst, in the measurement

373 uncertainty interval.

375 Secondly, empty freeze dryings were run in the Valduc Centre. They showed recovery of 376 water with a significant specific activity. The results are presented in Table 1.

Table 1: Mass and specific activity of water recovered after empty freeze dryings performed in the Valduc Centre

\begin{tabular}{|c|c|c|}
\hline $\begin{array}{c}\text { Duration of freeze drying } \\
(\mathrm{h})\end{array}$ & $\begin{array}{c}\text { Specific activity of the } \\
\text { Mass of recovered water }(\mathrm{g})\end{array}$ & 200 \\
\hline 168 & 0.4 & No measurement \\
\hline 168 & 0.0 & 257 \\
\hline 146 & 0.8 & \\
\hline
\end{tabular}

380

381 The results of the first and third freeze dryings fit well with the results presented in Fig. 4. For

382 example, $0.8 \mathrm{~mL}$ of "parasite" water with a specific activity of $257 \mathrm{~Bq} \mathrm{~L}{ }^{-1}$ can explain an

383 overestimation of the specific activity of about $+4 \mathrm{~Bq} \mathrm{~L}^{-1}$ in a sample of $47.5 \mathrm{~mL}$ in which the

384 specific activity is about $23 \mathrm{~Bq} \mathrm{~L}^{-1}$.

386 Nevertheless, the mass of water collected during freeze drying appears variable, as does its

387 specific activity. This indicates that an intermittent mechanism (most probably depending on

388 atmospheric conditions) leads to the pollution phenomenon. 
390 In the third repetition, the specific activity of the recovered water was about $257 \mathrm{~Bq} \mathrm{~L}^{-1}$ where

391 the specific activity of atmospheric vapour measured by bubbling during freeze drying was

392 only $90.8 \mathrm{~Bq} \mathrm{~L}^{-1}$. This means in our opinion that, in addition to a possible leak in the

393 apparatus (before the cold trap), four other ways of contamination might be suspected: as the sample is frozen before freeze drying, atmospheric water is able to condense at its surface in the time lapse between storage and introduction into the freeze drying system; the water vapour in the air present in the freeze drying system (about 6 L in the case of the Heto Drywinner PL 3000) is prone to condense starting from the moment when the cold trap is at its set-point temperature to the instant when vacuum is established in the system; the water vapour in air that fills the freeze drying system when the vacuum is broken at the end of freeze drying can also condense in the cold trap; some exchanges may occur from the atmosphere to the condensed water while the water melts in the cold trap for recovery.

It appears that during the different steps of a freeze drying process, some atmospheric water can be condensed or exchange and therefore be mixed to the extracted free water of the samples. The efficiency measured in water recovery is of the order of $97 \%$. The mass of external water cannot be identified in the different tests because it surely compensates sample water which is lost at the same time. This phenomenon was fortunately discovered due to the ambiance in the Valduc Centre that is sufficiently tritiated to be measured. Nevertheless, 
414 tritiated atmosphere. In that case, the water extracted would be "demarked" with non-tritiated

415 water, even if it is within the uncertainty interval of measure.

416

417 One way to limit the deviation due to this pollution of the extracted water would be to freeze

418 dry larger samples to dilute the effect in the water extracted from the sample or to operate

419 freeze drying in a dry atmosphere.

420

\subsection{Influence of the mass of sample in distillation techniques}

The free water of nineteen aliquots from the same milk sample was extracted by distillation

using a rotating evaporator or a distillation bridge. The results of this series of experiments are presented in Fig. 6. As expected, the nineteen specific activities measured are well described by a Gaussian distribution. Uncertainties ranged from $14 \%$ for higher specific activities to $17 \%$ for lower specific activities.

Fig. 6: Specific activity of extracted water as a function of the mass of treated samples and normality of the distribution of density of measured specific activities

Small samples $(<60 \mathrm{~g})$ and large samples $(\geq 60 \mathrm{~g})$ present almost the same average value, respectively $22.3 \mathrm{~Bq} \mathrm{~L}^{-1}$ and $22.8 \mathrm{~Bq} \mathrm{~L}^{-1}$, but different standard deviations: $2.17 \mathrm{~Bq} \mathrm{~L}^{-1}$ and

$0.88 \mathrm{~Bq} \mathrm{~L}^{-1}$. The variation of these average values may be explained by differences in the proportions of water extracted in each case (respectively $84.4 \%$ vs. $85.9 \%$ ).

437 Treating very large samples (> $100 \mathrm{~g}$ ) is not easy: experimentally we observe that the milk is 438 boiling and spurting very rapidly after the beginning of each repetition. This phenomenon is 439 prone to contaminate both extracted and condensed water. Treating small samples allowed the 
use of larger vessels compared to the sample volume: a difference of a factor of 5 between the

441 volumes of the Erlenmeyer flask and the sample is sufficient to limit spurting.

442 Each distillation technique also showed its own limitations with regard to the mass of the

443 samples treated.

Fig. 7: Proportion of water extracted (in \% of weight of total sample) versus mass of sample treated. The mean a forced-air oven at $102^{\circ} \mathrm{C}$.

While the rotating evaporator efficiently dehydrates only samples between $100 \mathrm{~g}$ and $400 \mathrm{~g}$, was not complete as it was interrupted to soon) (Fig. 7).

In these experiments, distillations of 60-100 g milk samples under reduced pressure using a activities.

\subsection{Influence of the state of dehydration}

The specific activities measured were viewed with respect to the state in which each increase slightly with the degree of dehydration but neither Spearman's nor Student's correlation tests revealed a correlation between the two parameters. 
Further experiments were then carried out with sequential distillations performed on different milks. The results are shown in Fig. 9 and Fig. 10. To facilitate comparison of the results, specific activities are expressed as relative activities ( 1 corresponds to the mean specific activity at the end of each sequential distillation) and levels of water extraction are expressed as a $\%$ of the total mass of sample at the end of each distillation. In Fig. 9 the specific activity 471 of each aliquot seems to follow a two-component function.

472

Fig. 9 : Specific activity of aliquots of extracted water versus the proportion of water extracted

474 The line plots the variation modelled with equation (11) with $\mathrm{p}=0.04, \alpha=1.14$, and $\beta=11.7$. Each type of label represents a set of repetition. Four repetitions were performed on one sample of milk (squares), two on a second sample (triangles) and lozenges represent a set performed on a third sample. Mean specific activity of extracted water $=1$.

Fig. 10 : Mean specific activity versus proportion of water extracted. The solid line was computed using relation (10) with $p=0.04, \alpha=1.14$, and $\beta=11.7$. Each type of label represents a repetition set. Four repetitions were performed on one sample of milk (squares), two on a second (triangles) and lozenges represent a set performed on a third. Mean specific activity of extracted water $=1$

Isotopic fractionation during distillation is generally described by means of the Rayleigh 1902).

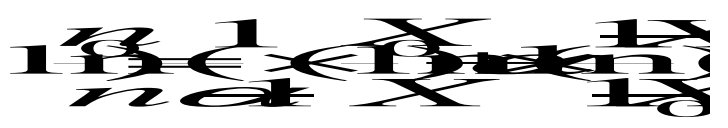

where:

$\mathrm{X}_{0}$ : is the initial mole fraction of HTO in the sample 
494

495 Kim and Baumgärtner (1997) reported that tritium enrichment on distillation of pure

$\mathrm{HTO} / \mathrm{H}_{2} \mathrm{O}$ can be calculated using the Rayleigh formula taken under the following form (3):

$$
A V_{r}=\Delta V_{o}\left(\frac{V_{0}}{V_{r}}\right)^{-\frac{1}{a}}
$$

498 Where:

499

500

501

502

503

504

505

506

$$
V P I E=\frac{P_{\mathrm{H}_{2} \mathrm{O}}}{P_{\text {HTO }}} \approx \alpha=\frac{\left(X_{T} / X_{H}\right)_{L}}{\left(X_{T} / X_{H}\right)_{V}}
$$


$514 \mathrm{P}_{\mathrm{H} 2 \mathrm{O}}$ and $\mathrm{P}_{\mathrm{HTO}}$ are the vapour pressures of pure water and pure tritiated water, respectively,

$515 \mathrm{X}_{\mathrm{T}}$ and $\mathrm{X}_{\mathrm{H}}$ stand respectively for the molar fractions of HTO and water, in the liquid (L) and $516 \quad \operatorname{vapour}(\mathrm{V})$ phase.

518 In order to fit with our experiments, residual volume $\left(\mathrm{V}_{\mathrm{r}}\right)$ and specific activity $\left(\mathrm{A}_{\mathrm{r}}\right)$ were 519 replaced in (3) by extracted volume $\left(\mathrm{V}_{\mathrm{e}}\right)$ and specific activity $\left(\mathrm{A}_{\mathrm{e}}\right)$ using relations (5) and (6). 520 Equation (7) is thus obtained:

521

522

523

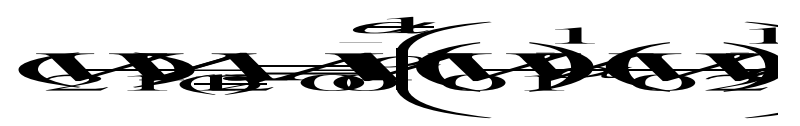

By considering (3):

$$
V_{e} A_{e}=A_{0} V_{0}\left(1-\left(\frac{V_{0}}{\left(V_{0}-V_{e}\right)}\right)^{\frac{-1}{\alpha}}\right)
$$
beginning and at the end of the extraction of the given aliquot (8):

Lastly, each aliquot sampled during the distillation has a specific activity $\left(\mathrm{A}_{(1-2)}\right)$ which is the mean value of (7) between $V_{1}$ and $V_{2}$, respectively the volume of water extracted at the

$536 \quad$ theoretical plate (HETP) and thus $\alpha \square$ (Fukada, 2004) $\square$ 
538 Fig. 11 : Extraction of $\mathrm{HTO}$ during distillation of pure $\mathrm{HTO} / \mathrm{H}_{2} \mathrm{O}$ versus proportion of free water extracted

Lines are calculated by equations (7) and (9). Triangles and circles correspond to experimental values. Mean specific activity of extracted water $=1$

Conversely, with samples of milk, at percentages of free water extracted higher than $\sim 95 \%$,

(7) and (8) generally fail at modelling the observed experimental behaviour of the relative

activity of extracted water, due to the drastic increase in relative activity measured at the end

of the water extraction process (Fig. 9). When most of the water is extracted from the milk, obtained in two main ways: a complete theoretical description of the sources of non-ideality or a blind parameterization of the observed effect. As for the theoretical description, in its simplest form a model may at least take account of two kinds of water, cosphere (hydration) retains the properties of the pure solvent (Jancso and Van Hook, 1974). In this part of the study, we will try to parameterize the observed effect and confine our work to the consequences of this behaviour on the metrology of tritium specific activity.

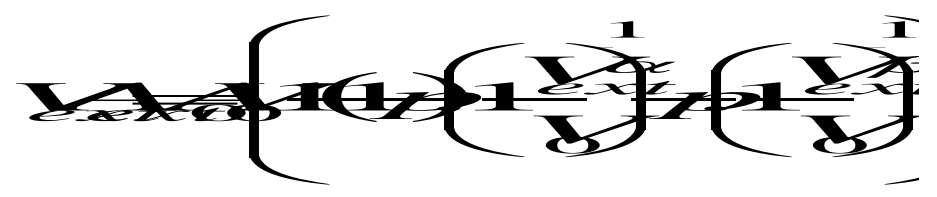

The specific activity of an aliquot is thus given by (10):

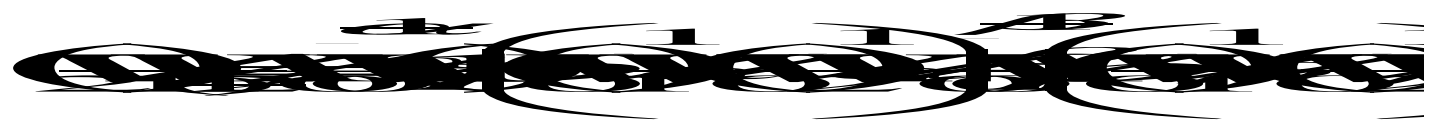


563 The experimental data presented in Fig. 9 can be modelled using relation (10). The best

564 values for $p, \alpha$, and $\beta$ (which are respectively $0.04,1.14,11.7$ ) were estimated by a function in

565 the R software (R Core Team, 2012) which carries out minimization of a function (f) using a

566 Newton-type algorithm. In R software, this function is called nlm. Each first aliquot of the

567 different repetitions has a variable specific activity as compared to the mean final specific

568 activity of the set. This is most probably an artefact linked to the experimental conditions. It is

569 noticeable in Fig. 10 that this value has a perceptible impact on the mean specific activity of,

570 say the 4-5 first aliquots. Using the model based on relation (9) we learn that even with a

571 (hypothetically) perfect dehydration apparatus, if the dehydration is interrupted when $10 \%$ of

572 water remains (a situation that may happen if the temperature is too low, the pressure too high

573 or the dehydration simply is uncompleted); the measured specific activity of the extracted

574 water should thus be underestimated by about $9 \%$.

\subsection{The dead volume, an example of a combination of sources of biases}

In light samples (ranging from $15 \mathrm{~g}$ to $60 \mathrm{~g}$ ), the measured specific activities of water extracted with a rotating evaporator were systematically lower than those obtained with a distillation bridge. Additional experiments were performed to explain this particular point. The rotating evaporator condenser indeed has a dead volume estimated to be $7.0 \mathrm{~mL} \pm 1.2$ $\mathrm{mL}$. In other words, $7 \mathrm{~mL}$ must reach the condenser before the first drop of distillate is observed and $7 \mathrm{~mL}$ remain in the condenser at the end of distillation. A model of the timecourse of the specific activities in the condenser and in the distillate during distillation based on an isotopic fractionation was set up.

587 Let us now consider the following assumptions as axiomatic: 
- $\quad$ as described above isotopic fractionation exists,

- $\quad$ in the condenser of the rotating evaporator, equilibrium between vapour and condensed phase is instantaneous, the same mass of condensed vapour to the distillate.

593

594 Now, introducing the subscript "cond." to denote the activity (or the mass) in the condenser; 595 from the beginning of the distillation to the extraction of a mass of water equal to the mass of water in the dead volume, we have:

597

$$
A_{\text {conid }}=A_{e x t}
$$

As soon as the dead volume of the condenser is filled:

601

602

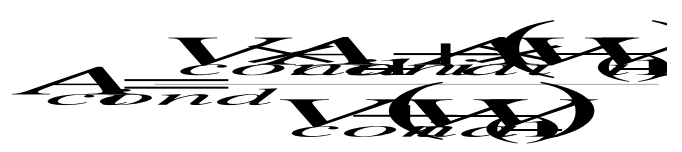

603

604 We consider that no water flows into the distillate until the dead volume of the condenser is 605 full and introduce the subscript "dist." to denote a specific activity of the distillate, thus:

606

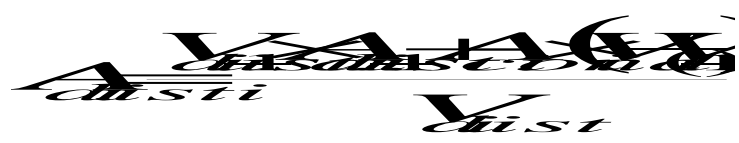

609 This model was tested on the different data sets of our results as well as with an experiment

610 where the condenser was first saturated with untritiated water before distilling tritiated water.

611 All sets of results are well-fitted by the present model. It shows how the measured specific 
612 activity (measured in the distillate) is modified by the loss of water in the dead volume (Fig.

613 12) for a known dead volume (7 $\mathrm{mL}$ in this example).

614

615

Fig. 12: Modelled evolution of specific activity of water in different compartments during the distillation of a $20 \mathrm{~g}$ sample of milk (Mean specific activity of extracted water $=1$ ).

617

618 This influence depends on the mass of the sample and on its specific activity (Fig. 13). For 619 samples of milk lower than $10 \mathrm{~g}$, the bias is less than $3 \%$. Actually, as there is only $8.7 \mathrm{~g}$ of 620 water in $10 \mathrm{~g}$ of milk and the dead volume of the condenser being estimated to $7 \mathrm{~mL}(7 \mathrm{~g})$, the 621 few drops that flow out from the condenser has a specific activity that is fully representative 622 of water extracted from the sample. For samples with $15 \mathrm{~g} \leq \mathrm{M} \leq 75 \mathrm{~g}$, the underestimation of 623 the specific activity is about $-6 \%$. The latter is in good agreement with the results presented 624 in Fig. 8 which represents how specific activities of different samples are distributed as a 625 function of their final rate of dehydration. Lastly, using samples larger than $200 \mathrm{~g}$ is a necessary condition to obtain a deviation that remains below $3 \%$ when using a rotating evaporator.

628

Fig. 13: Bias due to the rotating evaporator versus mass of sample. The model described above was tested with 630 different masses of sample (from $7 \mathrm{~g}$ to $10000 \mathrm{~g}$ ) to determine how the modelled bias induced by the dead

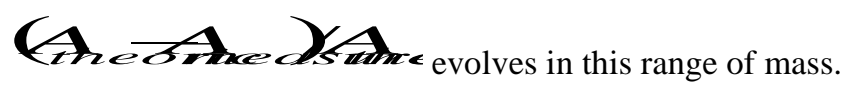

\section{Conclusion}

634

635 In this study we show that each methodological aspect tested (water removal technique, mass

636 of sample and final state of dehydration) is able to induce a bias in the specific activity

637 measured in the extracted water. In most environmental monitoring situations, these biases 
638 remain close to the uncertainty of measurement when liquid scintillation is used in the usual 639 conditions (i.e. about $15 \%$ when measuring a sample of $10 \mathrm{~mL}$ with an activity of $20 \mathrm{~Bq} . \mathrm{L}^{-1}$

640 water mixed with $10 \mathrm{~mL}$ of UltimaGold LLT (Pointurier et al., 2003) for 200 minutes).

641 Nevertheless, some of these biases can produce systematic underestimations of the actual 642 specific activity. First, it was shown that in the conditions of this study the water extracted by

643 freeze drying had a specific activity significantly higher than the water extracted using a 644 distillation technique based on the same milk sample. This is explained by a pollution of the 645 extracted water by atmospheric water before, after or during the freeze drying process. This 646 particular behaviour became apparent due to the fact that atmospheric water in the Valduc 647 centre is has slightly elevated ambient levels of tritium. Caution must thus be taken to avoid 648 marking during freeze drying, especially when the specific activity of the sample is not of the 649 same order of magnitude as the specific activity of the atmospheric vapour. An easy and 650 economic way to prevent this phenomenon would be to freeze dry larger samples (at least 100 $651 \mathrm{~g})$ which would dilute the effect of atmospheric water condensation. A more suitable way 652 nevertheless would be to operate freeze drying in a dry atmosphere and to break the vacuum 653 with dry gas.

654

655 When dehydration is performed by distillation under reduced pressure, the dead volumes of 656 the devices (especially those of the condenser) have to be limited as they can induce a bias in 657 the estimation of the mass of extracted water as well as and in the measurement of specific 658 activities. It appears that using a distillation bridge suits a wider range of samples masses than 659 a rotating evaporator, the latter should be preferred for large samples (> $300 \mathrm{~mL})$.

660

661 Lastly, in this study a fractionation effect during dehydration proved to be more significant 662 than expected. This shows the necessity to perform dehydration until there is no water left in 
663 the sample. When a fraction of water cannot be extracted without taking the risk of damaging

664 the dry matter, the residual fraction of water should be estimated and the specific activity measured corrected using formula (9) proposed in this work.

666

667 The effects of the different sources of biases must be summed. For instance, if a distillation

668 using a rotating evaporator (dead volume of $7 \mathrm{~mL}$ ) of a sample of $20 \mathrm{~mL}$ of milk is

669 interrupted when $10 \%$ of water remains in the matrix, the measured specific activity would

670 be about $91 \%$ of the real specific activity and the standard deviation of this result would be

$67111 \%$ additionally increase by the uncertainty of measure (about $15 \%$ in usual conditions).

672 Overall, this would lead to a global underestimation of about $10 \%$ and an uncertainty of \pm 26

$673 \%$.

674

675 In the literature, VPIE has been shown to decrease while temperature increases without

676 differences being measured between vaporization and sublimation (Baumgärtner and Kim,

677 1990). It has also been shown that a link exists between isotopic fractionation during

678 extraction of water and a three-layer model for bound water (Kim and Baumgärtner, 1997)

679 (described by Drost-Hansen). The results gathered herein using milk samples show

680 fractionation behaviour which can be described by a two-component formula, each

681 component being based on specific Rayleigh distillation processes. These results bring to

682 mind the features of two fractions of water that coexist in milk: the first one (96\%) that acts as

683 pure water (free water) and the second (4\%) which presents an isotopic separation factor $\beta$

684 equal to 11.7 much higher than that of pure water; $\alpha=1.14$. The fraction described here by

685 the isotope separation factor $\beta$ may be bound water.

686

687 Acknowledgements 
688 The authors would like to thank to the Conseil Régional de Bourgogne (France) for the 689 financial support of this study. 
691 Baumgärtner F, Kim MA. Isotope effects in the equilibrium and non-equilibrium vaporization

Boyer C, Vichot L, Fromm M, Losset Y, Tatin-Froux F, Guétat P, et al. Tritium in plants: a review of current knowledge. Environmental and experimental botany 2009; 67: 3451.

Diabaté S, Strack S. Organically bound tritium. Health physics. 65, 1993, pp. 698-712.

Fukada S. Tritium isotope separation by water distillation column packed with silica-gel beads. Journal of Nuclear science and technology 2004; 41: 619-623.

Guétat P, Douche C, Hubinois JC. Tritium and the environment: Sources, measurement and transfer. In: commission E, editor. EU Scientific seminar 2007 "Emerging issues on tritium and low energy beta emitters". 152, Luxembourg, 2008, pp. 59-72.

IRSN/DEI. Le tritium dans l'environnement - Synthèse des connaissances. Tritium-Livre blanc, 2010, pp. 44-110.

Jacobs DG. Sources of tritium and its behaviour upon release to the environment. In: D-24635 T, editor. U.S. Atomic Energy Commission/Division of Technical information, Oak Ridge, Tennessee, 1968.

Jancso G, Van Hook WA. Condensed phase isotope effects (especially vapor pressure isotope effects). Chem. Rev. 1974; 74: 689-750.

Kakiuchi M. Distribution of isotopic water molecules, $\mathrm{H}_{2} \mathrm{O}$, $\mathrm{HDO}$ and $\mathrm{D}_{2} \mathrm{O}$, in vapor and liquid phase in pure water and aqueous solution systems. Geochimica et Cosmochimica Acta 2000; 64: 1485-1492.

Kim MA, Baumgärtner F. Tritium fractionation in biological systems and in analytical procedures. Radiochimica Acta 1991; 54: 121-128.

Kim MA, Baumgärtner F. Tritium fractionation in anomalous water bound to environmental samples. Journal of Environmental Radioactivty 1997; 36: 111-127.

Pointurier F, Baglan N, Alanic G, Chiappini R. Determination of organically bound tritium background level in biological samples from a wide area in the south-west of France. Journal of environmental radioactvity 2003; 68: 171-189.

Rayleigh L, Strutt JW. On the distillation of binary mixtures. Philosophical magazine 1902; 4: 521-537.

R Core Team. R: A language and environment for statistical computing. R Foundation for Statistical Computing, Vienna, Austria, 2012.

UNSCEAR. Sources and effects of ionizing radiation (Report to the General Assembly). 1. UN, New York, 2008. 
740 Van Hook WA. Vapor pressures of the isotopic waters and ices. The journal of physical chemistry 1968; 72: 1234-1244.

Weaver CL, Harward ED, H. T. Peterson J. Tritium in the environment from Nuclear Powerplants. Public Health Reports 1969; 84: 363-371.

Wood MJ, McElroy RGC, Surette RA, Brown RM. Tritium sampling and measurement. Health physics 1993; 65: 610-627. 